\title{
O059: Introducing an intervention bundle to reduce the incidence of catheter related infections
}

\author{
E Smid, J Wille*, S Greeff de, T Hopmans, M Koek \\ From 2nd International Conference on Prevention and Infection Control (ICPIC 2013) \\ Geneva, Switzerland. 25-28 June 2013
}

\begin{abstract}
Introduction
Literature indicates that the incidence of catheter related infections can be reduced after implementing a so-called intervention bundle. In line with this, an intervention bundle to prevent catheter related infections was introduced in The Netherlands, aiming (1) at a 90\% compliance with the bundle and (2) to reduce the incidence of catheter related infections.
\end{abstract}

\section{Objectives}

To assess whether a 90\% compliance with the bundle and/or a reduction in the incidence of catheter related infections in Dutch hospitals to less than three cases of infection per 1000 catheter-days have been established.

\section{Methods}

In 2009 an intervention bundle consisting of six items was introduced. The six items were hand hygiene, precautions during insertion, cleaning the skin, selection insertion site, daily check on indication, and daily check on insertion site. All Dutch hospitals were asked to register the compliance with the intervention bundle together with the incidence of infections for all central venous catheters. Registration took place from January 2009 until December 2012. The intervention bundle is analysed on registration and compliance level.

\section{Results}

64 from the 93 hospitals in The Netherlands registered for participation, 31 hospitals registered infection-data and 27 registered data about the intervention bundle. Registration of the complete bundle increased from $7 \%$ in 2009 to $61 \%$ in 2012. During these four years compliance with the bundle increased from $76 \%$ to $87 \%$. In 2009, 6 out of the 9 registering hospitals (67\%) had an incidence of less than three cases of infection per 1000 catheter-days. In 2012, 20 out of 25 registering hospitals $(80 \%)$ had less than three infections per 1000 catheter-days. Four hospitals in 2012 (16\%) had more than five infections per 1000 catheter-days.

\section{Conclusion}

Compliance with the complete bundle increased over the four years to $87 \%$, but the target of $90 \%$ is not yet met. In 2012 a higher proportion of the hospitals had an incidence of less than three infections per 1000 catheterdays (not significantly different), but still some hospitals had more than five infections per 1000 catheter-days. We conclude that for both compliance and infectionrates there is still room for improvement.

\section{Disclosure of interest}

None declared.

Published: 20 June 2013

doi:10.1186/2047-2994-2-S1-059

Cite this article as: Smid et al:: O059: Introducing an intervention bundle to reduce the incidence of catheter related infections. Antimicrobial Resistance and Infection Control 2013 2(Suppl 1):059.

Clb/PREZIES, RIVM, Bilthoven, The Netherlands

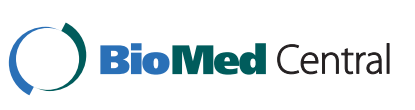

() 2013 Smid et al; licensee BioMed Central Ltd. This is an Open Access article distributed under the terms of the Creative Commons Attribution License (http://creativecommons.org/licenses/by/2.0), which permits unrestricted use, distribution, and reproduction in any medium, provided the original work is properly cited. 